T. Adachi

Nagoya Math. J.

Vol. 110 (1988), 1-14

\title{
DISTRIBUTION OF CLOSED GEODESICS WITH A PREASSIGNED HOMOLOGY CLASS IN A NEGATIVELY GURVED MANIFOLD
}

\author{
TOSHIAKI ADACHI
}

\section{§1. Introduction}

Let $M$ be a compact Riemannian manifold whose geodesic flow $\varphi_{t}$ : $U M \rightarrow U M$ on the unit tangent bundle is of Anosov type. In this paper we count the number of $\varphi_{t}$-closed orbits and study the distribution of prime closed geodesics in a given homology class in $H_{1}(M, Z)$. Here a prime closed geodesic means an (oriented) image of a $\varphi_{t}$-closed orbit by the projection $p: U M \rightarrow M$.

In the preceding paper [3] with Sunada, we see that there exist infinitely many prime closed geodesics in each homology class. In this context we are led to a natural problem of the distribution of closed geodesics in a given homology class. Concerning this problem, Bowen [4], [5], having introduced a measure associated with closed orbits of an Axiom $A$ flow, showed that closed geodesics (without any restrictions on their homology classes) are uniformly equidistributed. Recently, Parry [8] provides an alternative proof for Bowen's result, based on the zeta functions of flows.

For a loop $c$ in $M$, let [c] be the homology class represented by $c$. Given a $\varphi_{t}$-periodic point $x \in U M$, we denote by $\mathfrak{p}(x)$ the closed orbit containing $x$.

Theorem 1. Let $M$ be a compact Riemannian manifold whose geodesic flow $\varphi_{t}$ is of Anosov type. For any $\alpha \in H_{1}(M, Z)$, the set of all $\varphi_{t}$-periodic points $x$ with $[p(\mathfrak{p}(x))]=\alpha$ is dense in $U M$.

In particular, prime closed geodesics in $\alpha$ form a dense subset in $M$.

To get more precise information on the distribution of prime closed

Received July $17,1985$.

Revised March 13, 1987. 
geodesics, we introduce the following orbital measures on $U M$. Given a coset $\mathscr{A}$ of a finite index subgroup of $H_{1}(M, Z)$ and a positive $T$ and $\varepsilon$, we define measures $\tilde{\mu}_{T, \varepsilon, s}$ and $\tilde{\nu}_{T, \varepsilon, s}$ by

$$
\begin{aligned}
& \tilde{\mu}_{T, \varepsilon, \infty}(g)=\sum_{\substack{\mathfrak{p}=\mathfrak{p}(x),[\mathcal{p}(\mathfrak{p})] \in \mathscr{A} \\
T-\varepsilon<\tau(\mathfrak{p})<T+\varepsilon}} \int_{0}^{\tau(\mathfrak{p})} g\left(\varphi_{t}(x)\right) d t / \tau(\mathfrak{p}), \\
& \tilde{\nu}_{T, \varepsilon, \infty}(g)=\sum_{\substack{p=p(x),[p(p)] \in \mathscr{A} \\
T-\varepsilon<\tau(\mathcal{p})<T+\varepsilon}} \int_{0}^{\tau(\mathfrak{p})} g\left(\varphi_{t}(x)\right) d t,
\end{aligned}
$$

for each smooth function $g$ on $U M$, where $\tau(\mathfrak{p})$ denotes the period of $\mathfrak{p}$. Normalize them so that

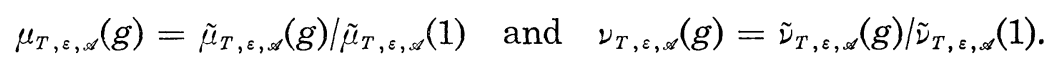

TheOREm 2. Under the same assumption as in Theorem 1, prime closed geodesics with a preassigned homology class are "nearly" equidistributed in the following sense: For any cosets $\mathscr{A}, \mathscr{B}$ of an arbitrary finite index subgroup of $H_{1}(M, Z)$, positive $\varepsilon$ and a smooth function $g$,

$$
\begin{aligned}
& \nu_{T, \varepsilon, \mathscr{A}}(g) \longrightarrow \int_{U M} g d \mu, \quad \nu_{T, \varepsilon, \mathscr{A}}(g) / \nu_{T, \varepsilon, \mathscr{Q}}(g) \longrightarrow 1 \\
& \mu_{T, \varepsilon, \infty}(g) \longrightarrow \int_{U M} g d \mu, \quad \mu_{T, \varepsilon, \mathscr{A}}(g) / \mu_{T, \varepsilon, \mathscr{A}}(g) \longrightarrow 1
\end{aligned}
$$

as $T \rightarrow \infty$, where $\mu$ is the $\varphi_{t}$-invariant maximal entropy measure.

In the special case that $H_{1}(M, Z)$ is finite, we can conclude that prime closed geodesics are equidistributed.

Our approach to Theorem 1 is based on the technique of graphical approximations for flows, which was given in [3]. There is an irreducible oriented finite graph embedded in $U M$ such that each $\varphi_{t}$-closed orbit is approximated by a closed path in the graph (see §2). Briefly speaking, what we do is to compute traces associated with this graph. Edges are weighted so that they have information not only on periods of closed orbits but also on places where they are embedded. Once expressed by matrices the method given in [3] is refined in Section 4, in particular, Theorem 1 is proved. To prove Theorem 2 we apply a number-theoretic argument; we define "weighted" $L$-functions for $\varphi_{t}$ and analyze their analyticity and poles (cf. [2], [8], [9]).

The author is very grateful to T. Sunada for giving him much valuable advice. 


\section{§ 2. Graphical approximation of the geodesic flow}

In this section we recall a graphical approximation of the geodesic flow $\varphi_{t}$ given in [3], which is a consequence of Bowen's symbolic dynamics $[6]$.

We first sketch some basic materials. An oriented finite graph consists of a finite set of vertices $V$ and a set of edges $E \subset V \times V$. A path $c$ (or more precisely a $r$-step path) in a graph $(V, E)$ is an element of the form $c=\left(v_{0}, \cdots, v_{r}\right) \in V \times \cdots \times V$ (r+1 factor), with $\left(v_{i}, v_{i+1}\right) \in E$ for any $0 \leq i \leq r-1$. Put $o(c)=v_{0}, t(c)=v_{r}$ and $|c|=r$. We call $c$ closed if $o(c)=t(c)$, and call prime if it is not a non-trivial tower of other closed path. Two $r$-step closed paths $c=\left(v_{0}, \cdots, v_{r}\right)$ and $c^{\prime}=\left(w_{0}, \cdots, w_{r}\right)$ are said to be equivalent if $v_{i+k}=w_{i}, i \in Z / r Z$ for some $k$. A cycle $\langle c\rangle$ is the equivalence class containing $c$. If $c$ is a $r$-step closed path, there are at most $r$ different closed paths in $\langle c\rangle$. From now we only treat irreducible oriented finite graphs, that means for any two vertices there exists a path joining them.

Given a finite graph $(V, E)$, a subshift of finite type $\Sigma(V, E)$ is a compact 0-dimensional space defined by

$$
\left\{\xi=\left(\xi_{i}\right) \in \prod_{Z} V \mid\left(\xi_{i}, \xi_{i+1}\right) \in E \quad \text { for any } i \in Z\right\} .
$$

The shift operator $\sigma: \Sigma(V, E) \rightarrow \Sigma(V, E)$ is a continuous map defined by $\sigma(\xi)_{i}=\sigma(\xi)_{i+1}$. For a strictly positive function $l$ on $\Sigma(V, E)$ we define a suspension $\Sigma(V, E, l)$ by

$$
\{(\xi, s) \mid \xi \in \Sigma(V, E) \text { and } 0 \leq s \leq l(\xi)\},
$$

where $(\xi, l(\xi))$ and $(\sigma \xi, 0)$ are identified. On this space there is a vertical flow $\sigma(l)_{t}$ given by $\sigma(l)_{t}(\xi, s)=(\xi, t+s)$ with appropriate identifications.

Let $c=\left(v_{0}, \cdots, v_{r}\right)$ be a closed path in $(V, E)$. Define $\xi(c) \in \Sigma(V, E)$ by $\xi(c)_{j}=v_{i}, j \equiv i(\bmod r)$, and put

$$
\begin{aligned}
\mathfrak{p}(c) & =\left\langle\sigma(l)_{t}(\xi(c), 0) \mid 0 \leq t \leq \tau(c)\right\rangle, \\
\tau(c) & =\sum_{i=0}^{r-1} l\left(\sigma^{i} \xi(c)\right) .
\end{aligned}
$$

We frequently identify a cycle $\langle c\rangle$ with the $\sigma(l)_{t}$-orbit cycle $\mathfrak{p}(c)$. Here to avoid any confusion we explain the meaning of orbit cycles. An orbit cycle $\mathfrak{p}$ is a $k$-th tower of some closed orbit $\mathfrak{p}^{\prime}$, and the period of $\mathfrak{p}$ is $k$ times the minimal period of $\mathfrak{p}^{\prime}$. A closed orbit is also called a prime orbit cycle. 
Let us return to our geodesic flow. The technique of graphical approximations is based on a Markov family. Each Markov family $V$ defines an irreducible non-circuit graph $(V, E)$, which is naturally embedded in $U M$ as a $C W$-complex. The importance of this graph is the following relationship with the flow $\varphi_{t}$ : There are strictly positive Lipschitz continuous function $l$ and a continuous surjective finite-to-one map $\rho: \Sigma(V, E, l)$ $\rightarrow U M$ such that $\rho \circ \sigma(l)_{t}=\varphi_{t} \circ \rho$. We refer to this suspension as a principal suspension. Let c denote the natural embedding of $(V, E)$ into $U M$. It is known [3] that if we define a finite-to-one correspondence $\Phi$ of the set of cycles in $(V, E)$ onto the set of $\varphi_{t}$-orbit cycles by $c \mapsto \rho(\mathfrak{p}(c))$ then $\iota(c)$ and $\Phi(\langle c\rangle)$ are free homotopic. Therefore we can reduce the problem of estimating the number of $\varphi_{t}$-closed orbits to that of counting prime closed paths in an irreducible finite graph associated with $\varphi_{t}$ (see [3]).

Among various features of geodesic flows we pay attention to the reversible property; $\varphi_{t}(-x)=-\varphi_{-t}(x)$ for any $x \in U M$. One can construct a Markov family and the principal suspension so that they inherit this property (see [1] and [14]).

Proposition 3. There exists a Markov family $(V, E)$ for $\varphi_{t}$ of arbitrarily small size and with orientation-reversing involution $\kappa: V \rightarrow V$ such that for any closed path $c=\left(v_{0}, \cdots, v_{r}\right), p(\Phi(\langle c\rangle))$ and $p(\Phi(\langle\kappa c\rangle))$ represent the same closed geodesic in $M$ with different orientations, where $\kappa c=\left(\kappa v_{r}, \cdots, \kappa v_{0}\right)$.

\section{$\S 3$. Twisted Perron-Frobenius theorem}

In counting closed paths in a graph, we shall make use of "twisted" aperiodic matrices associated to this graph. We summarize in this section some of results about eigenvalues of "twisted" non-negative matrices. See [3] for proofs.

Let $(\boldsymbol{V}, \boldsymbol{E})$ be an oriented irreducible finite graph. Regarding $(\boldsymbol{V}, \boldsymbol{E})$ as a 1-dimensional $C W$-complex, we denote by $\pi:(\hat{V}, \hat{E}) \rightarrow(V, E)$ the universal covering and by $\pi_{1}(V, E)$ the fundamental group, which is generated by homotopy classes of closed paths in $(V, E)$. Decompose $V$ into primitive parts $V_{1}, \cdots, V_{\nu}$ (see [2] for example), put $\hat{V}_{1}=\pi^{-1}\left(V_{1}\right)$ and choose a subset $\mathscr{D}$ in $\hat{V}_{1}$ so that $\hat{V}_{1}=\cup_{\gamma \in \pi_{1}} \gamma \mathscr{D}$ and $\gamma \mathscr{D} \cap \mathscr{D}=\varnothing$ if $\gamma \neq \mathrm{Id}$.

Given a character $\chi: \pi_{1}(\boldsymbol{V}, \boldsymbol{E}) \rightarrow\{\boldsymbol{z} \in \boldsymbol{C}|| \boldsymbol{z} \mid=1\}$, let $\mathscr{G}_{\chi}$ be the vector space of complex-valued functions $h$ on $\hat{V}_{1}$ satisfying $h \circ \gamma=\chi(\gamma) h$ for any $\gamma \in \pi_{1}(V, E)$. If one defines $\delta_{x, w}: \hat{V}_{1} \rightarrow C$ for $w \in \mathscr{D}$ by 


$$
\delta_{\chi, w}(v)= \begin{cases}\chi(\gamma) & \text { if } v=\gamma w \\ 0 & \text { otherwise }\end{cases}
$$

$\left\{\delta_{x, w}\right\}_{w \in \mathscr{D}}$ forms a basis of $\mathscr{G}_{\chi}$.

Let $F$ be a non-negative function on $E$. We always suppose $F \neq 0$. Define an operator $\mathscr{L}_{F, \chi}: \mathscr{G}_{\chi} \rightarrow \mathscr{G}_{\chi}$ by

$$
\mathscr{L}_{F, x} h(v)=\sum_{c} F\left(\pi\left(v_{0}\right), \pi\left(v_{1}\right)\right) \cdots F\left(\pi\left(v_{\nu-1}\right), \pi\left(v_{\nu}\right)\right) h\left(v_{\nu}\right),
$$

where $c=\left(v_{0}, \cdots, v_{\nu}\right)$ runs over all $\nu$-step closed paths in $(\hat{V}, \hat{E})$ with $o(c)=v$. By using the basis $\left\{\delta_{x, w}\right\}_{w \in \mathscr{P}}$, we represent $\mathscr{L}_{F, x}$ by a $d$-dimensional matrix $L_{F, \chi}$, where $d=\#\left(V_{1}\right)$, the cardinality of $V_{1}$. These matrices $L_{F, \chi}$ varies smoothly with respect to $F$ and continuously with respect to $\chi$.

When $\chi=1$, the trivial character, $L_{F, 1}$ is an aperiodic non-negative matrix. For a general $\chi$ we can view $L_{F, \chi}$ something like a matrix obtained by "twisting" $L_{F, 1}$ with the character $\chi$. Using Perron-Frobenius theorem we can prove

Proposition 4 ([3]). 1) $L_{F, 1}$ has a simple positive eigenvalue $\lambda(F)$ and an associated positive eigenvector,

2) other eigenvalue $\mu$ of $L_{F, 1}$ than $\lambda(F)$ satisfies $|\mu|<\lambda(F)$,

3) $\lambda(F)=\max _{h \in R^{d}}\left\{\min _{1 \leq j \leq d, h_{j} \neq 0}\left(L_{F, 1} h\right)_{j} / h_{j} \mid h \geq 0, h \neq 0\right\}$,

4) for each character $\chi$, any eigenvalue $\mu$ of $L_{F, x}$ satisfies $|\mu| \leq \lambda(F)$,

5) $L_{F, x}$ has an eigenvalue of the form $\lambda(F) \exp \sqrt{-1} \psi$ if and only if $\chi(\llbracket c \rrbracket)=\exp (\sqrt{-1} \psi ; c \mid / \nu)$ for any closed path $c$ in $(\boldsymbol{V}, \boldsymbol{E})$, where $\llbracket c \rrbracket$ is the conjugacy class in $\pi_{1}(V, E)$ represented by $c$.

Remark. The maximal eigenvalue $\lambda(F)$ does not depend on the choice of primitive part $V_{1}$.

\section{$\S 4$. Existence of infinitely many closed paths}

We shall prove the following proposition which is a key of this paper.

Proposition 5. Let $(V, E)$ be a irreducible finite graph of a Markov family for the geodesic flow $\varphi_{t}$ with associated involution $\kappa$. For each $\alpha \in$ $H_{1}(M, Z)$ and $v \in V$, there exist infinitely many prime closed paths $c$ in $(V, E)$ with $o(c)=v$ and with prime $\Phi(\langle c\rangle)$ satisfying $[p(\Phi(\langle c\rangle))]=\alpha$.

To prove this we utilize the irreducible graph $\left(V^{r}, E^{r}\right)$ of $r$-step paths in $(V, E)$, which is defined by 


$$
\begin{aligned}
& V^{r}=\left\{\boldsymbol{v}=\left(v_{0}, \cdots, v_{r}\right) \mid r \text {-step paths in }(V, E)\right\}, \\
& E^{r}=\left\{(\boldsymbol{v}, \boldsymbol{w}) \in V^{r} \times V^{r} \mid v_{i}=w_{i+1}, 0 \leq i \leq r-1\right\} .
\end{aligned}
$$

Note that this graph also admits an orientation reversing involution $\kappa$ : $V^{r} \rightarrow V^{r}$ given by $k\left(v_{0}, \cdots, v_{r}\right)=\left(\kappa\left(v_{r}\right), \cdots,\left(v_{0}\right)\right)$. The natural projection $\omega: V^{r} \rightarrow V$ defined by $\omega(\boldsymbol{v})=v_{0}$, though it also reverses the orientation, gives a connection between $\left(V^{r}, E^{r}\right)$ and $(V, E)$. For example, a closed path $c=\left(\boldsymbol{v}_{0}, \cdots, \boldsymbol{v}_{m}\right)$ in $\left(V^{r}, E^{r}\right)$ can be identified with the closed path $\omega(c)=\left(\omega\left(\boldsymbol{v}_{m}\right), \cdots, \omega\left(\boldsymbol{v}_{0}\right)\right)$ in $(V, E)$. Define a continuous map of $\left(V^{r}, E^{r}\right)$ into $U M$ by $\iota \circ \omega$ and a correspondence of the set of cycles in $\left(V^{r}, E^{r}\right)$ onto the set of $\varphi_{t}$-orbit cycles by $\Phi \circ \omega$. By Proposition 3, for any closed path $c, \iota \circ \omega(c)$ is free homotopic to $\Phi(\langle\omega c\rangle)$, and $p(\Phi(\langle\omega c\rangle))$ and $p(\Phi(\omega(\kappa c)\rangle))$ $=p(\Phi(\langle\kappa(\omega c)\rangle))$ represent the same closed geodesic with different orientations.

Approximating the princpal suspending function $\ell$ we have

Proposition 6 ([3]). Given a positive $\varepsilon$ with $\varepsilon<h_{\text {top }}$, there exist a positive integer $r$ and a strictly positive function $\tilde{f}$ on $E^{r}$ such that

1) $\lambda\left(\exp \left(\varepsilon-h_{\text {top }}(\tilde{f})\right)>1\right.$

2) $\min (\ell) \leq \tilde{f} \leq \max (\ell)$

3) $\tilde{f}(\kappa c)=\tilde{f}(c)$ for any closed path $c$ in $\left(V^{r}, E^{r}\right)$, where

$$
\tilde{f}(c)=\sum_{i=0}^{m-1} \tilde{f}\left(\boldsymbol{v}_{i}, \boldsymbol{v}_{i+1}\right) \quad \text { if } c=\left(\boldsymbol{v}_{0}, \cdots, \boldsymbol{v}_{m}\right) .
$$

Given $v \in V$, let $V_{1}^{r}$ be the primitive part of $V^{r}$ containing an element $\boldsymbol{v} \in V^{r}$ with $\omega(\boldsymbol{v})=v$. For simplicity we set $f=\left(\varepsilon-h_{\text {top }}\right) \tilde{f}$ and $\lambda=\lambda(\exp f)$. We define a non-negative function $g_{v}$ on $E^{r}$ by

$$
g_{v}(\boldsymbol{v}, \boldsymbol{w})= \begin{cases}1 & \text { if } \omega(\boldsymbol{w})=v \\ 0 & \text { otherwise }\end{cases}
$$

Let $\hat{H}_{1}=\hat{H}_{1}(M, Z)$ denote the character group of $H_{1}(M, Z)$. Regarding $\chi \in \hat{H}_{1}$ as a character of $\pi_{1}\left(V^{r}, E^{r}\right)$ through the homomorphisms

$$
\pi_{1}\left(V^{r}, E^{r}\right) \stackrel{\left\llcorner\circ \omega_{*}\right.}{\longrightarrow} \pi_{1}(U M) \stackrel{p_{*}^{*}}{\longrightarrow} \pi_{1}(M) \longrightarrow H_{1}(M, Z),
$$

we shall consider the matrices $L(\chi, s)=L_{\exp \left(f+s g_{v}\right), \chi}, s \in R$. Let $\lambda_{1}(\chi, s)$, $\cdots, \lambda_{d}(\chi, s)\left(\left|\lambda_{1}(\chi, s)\right| \geq \cdots \geq\left|\lambda_{d}(\chi, s)\right|\right)$ denote the eigenvalues of $L(\chi, s)$ and put $S_{m}(\chi, s)=$ Trace $\left(L(\chi, s)^{m}\right)$. By an easy calculation of matrix elements we have 


$$
S_{m}(\chi, s)=\sum_{c} \chi([c]) \exp \left\{f(c)+s g_{v}(c)\right\}:
$$

where $c$ runs over all $m \nu$-step closed paths in $\left(V^{r}, E^{r}\right)$ with $o(c) \in V_{1}^{r}$. The presence of the associated involution $\kappa$ guarantees that $S_{m}(\chi, 0)$ is a real number for any $m$ and $\chi \in \hat{H}_{1}$. In fact, we have

$$
\begin{aligned}
S_{m}(\chi, 0) & =\sum_{c} \chi([p \circ \iota \circ \omega(c)]) \exp f(c) \\
& =\sum_{c} \chi([p(\Phi(\langle\omega(c)\rangle))]) \exp f(c) \\
& =\sum_{c} \chi([-p(\Phi(\langle\kappa \omega(c)\rangle))]) \exp f(\kappa c) \\
& =\sum_{c} \overline{\chi([p(\Phi(\langle\kappa \omega(c)\rangle))])} \exp f(\kappa c)=\overline{S_{m}(\chi, 0)} .
\end{aligned}
$$

Hence $\lambda_{1}(\chi, 0) \in \boldsymbol{R}$ if $\left|\lambda_{1}(\chi, 0)\right|>\left|\lambda_{2}(\chi, 0)\right|$.

LEMMA 7. $\lambda(s)=\lambda_{1}(\mathbf{1}, s)$ is a smooth function on $\boldsymbol{R}$ with $\lambda^{\prime}(s)>0$.

Proof. Since $\lambda(s)$ is an isolated eigenvalue of $L(s)=L(\mathbf{1}, s)$, by perturbation theory of matrices, we know that $\lambda(s)$ is smooth and that there is a smooth map $u(s): \boldsymbol{R} \rightarrow \boldsymbol{R}^{d}$ with $L(s) u(s)=\lambda(s) u(s)$ and $u(s)>0$. It is an easy consequence of 3 ) of Proposition 4 that $\lambda^{\prime}(s) \geq 0$. Now suppose $\lambda^{\prime}\left(s_{0}\right)=0$ for some $s_{0}$. Put $C=\min _{1 \leq j \leq d}\left\{-u_{j}^{\prime}\left(s_{0}\right) / u_{j}\left(s_{0}\right)\right\}-1$ and define $h(s)=\exp \left\{C\left(s-s_{0}\right)\right\} u(s)$. Differentiating the equality $L(s) h(s)=\lambda(s) h(s)$, we have

$$
L^{\prime}\left(s_{0}\right) h\left(s_{0}\right)+L\left(s_{0}\right) h^{\prime}\left(s_{0}\right)=\lambda\left(s_{0}\right) h^{\prime}\left(s_{0}\right) .
$$

Since $h\left(s_{0}\right)>0$ and $L^{\prime}\left(s_{0}\right)=L_{g_{v} \exp \left(f+s_{0} g_{v}\right), 1}$ is a non-zero and non-negative matrix, we get

$$
L^{\prime}\left(s_{0}\right) h\left(s_{0}\right) \geq 0, \quad L^{\prime}\left(s_{0}\right) h\left(s_{0}\right) \neq 0, \quad L\left(s_{0}\right) h^{\prime}\left(s_{0}\right) \leq \lambda\left(s_{0}\right) h^{\prime}\left(s_{0}\right) .
$$

Again 3) of Proposition 4 implies

$$
L\left(s_{0}\right) h^{\prime}\left(s_{0}\right)=\lambda\left(s_{0}\right) h^{\prime}\left(s_{0}\right),
$$

which leads us to a contradiction $L^{\prime}\left(s_{0}\right) h\left(s_{0}\right)=0$.

For each $\alpha \in H_{1}(M, Z)$, we denote by $\mathscr{C}_{n}\left(V^{r} ; \alpha\right)$ the set of $n$-step closed paths $c$ in $\left(V^{r}, E^{r}\right)$ with $[p(\Phi\langle\omega c\rangle)]=\alpha$, and by $\mathscr{P}_{n}\left(V^{r} ; \alpha\right)$ the set of all prime $c \in \mathscr{C}_{n}\left(V^{r} ; \alpha\right)$ with prime $\Phi(\langle\omega c\rangle)$. Put

$$
\begin{aligned}
C_{m}(\alpha) & =\sum_{c \in \mathscr{C}_{m \nu}\left(V V_{;} ; \alpha\right), o(c) \in V_{1}^{r}} g_{v}(c) \exp f(c), \\
G_{m}(\alpha) & =\sum_{c \in \mathscr{P}_{m v}\left(V V_{r} ; \alpha\right), o(c) \in V_{1}^{r}} g_{v}(c) \exp f(c) .
\end{aligned}
$$


By the definition of $g_{v}$, to prove Proposition 5, it is enough to show $G_{m}(\alpha)>0$ for infinitely many $m$.

Let $d \chi$ denote the Haar measure on $\hat{H}_{1}$ with $\int_{\hat{H}_{1}} d \chi=1$. Using the orthogonal relation of characters and (1), we have

$$
C_{m}(\alpha)=\int_{\hat{H}_{1}} \chi(-\alpha) S_{m}^{\prime}(\chi, 0) d \chi,
$$

where $S_{m}^{\prime}(\chi, 0)=\left.(d / d s)\right|_{s=0} S_{m}(\chi, s)$. Since $f$ and $g_{v}$ are real-valued, one can rewrite this equality as follows;

$$
C_{m}(\alpha)=\int_{\hat{H}_{1}} \mathscr{R} e\left\{\chi(-\alpha) S_{m}^{\prime}(\chi, 0)\right\} d \chi .
$$

In order to estimate $C_{m}(\alpha)$ we shall deal with eigenvalues of the matrix $L(\chi, s)$. By Proposition $4, L(\chi, s)$ has an eigenvalue of the form $\lambda_{1}(\chi, s)=\exp (\sqrt{-1} \psi) \lambda(s), 0 \leq \psi<2 \pi$, if and only if

$$
\chi(\llbracket c \rrbracket)=\exp (\sqrt{-1} \psi|c| / \nu)
$$

for any closed path $c$ in $\left(V^{r}, E^{r}\right)$. As we have

$$
\chi(\llbracket c \rrbracket)=\exp (\sqrt{-1} \psi|\kappa c| / \nu)=\chi(\llbracket \kappa c \rrbracket)=\overline{\chi(\llbracket c \rrbracket)},
$$

such case occurs if and only if $\chi=1$ or $\chi=\chi_{0}$, where $\chi_{0} \in \hat{H}_{1}$ (if exists) uniquely determined by the formula

$$
\left.\chi_{0}(\llbracket c]\right)=\exp (\sqrt{-1}|c| / \nu)
$$

for any closed path $c$ in $\left(V^{r}, E^{r}\right)$.

Given a positive $B$ with $B<\lambda$, we set

$$
\begin{aligned}
& U_{B}=\left\{\chi \in \hat{H}_{1}|| \lambda_{1}(\chi, 0) \mid>B\right\}, \\
& U_{B}^{+}=\left\{\chi \in \hat{H}_{1} \mid \lambda_{1}(\chi, 0)>B\right\},
\end{aligned}
$$

and

$$
U_{B}^{-}=\left\{\chi \in \hat{H}_{1} \mid \lambda_{1}(\chi, 0)<-B\right\} .
$$

By petturbation theory of eigenvalues, if $B$ is sufficiently close to $\lambda$, we have $\left|\lambda_{1}(\chi, 0)\right|>\left|\lambda_{2}(\chi, 0)\right|$. Hence $U_{B}=U_{B}^{+} \cup U_{B}^{-}$, and $U_{B}^{+}$and $U_{B}^{-}$are open neighborhoods of 1 and $\chi_{0}$ respectively. It should be also noted that $U_{B}^{+}$ and $U_{B}^{-}$shrink to $\{\mathbf{1}\}$ and $\left\{\chi_{0}\right\}$ as $B$ goes to $\lambda$.

We now estimate $C_{m}(\alpha)$ from below.

LEMma 8. There is a constant $C_{1}$ such that if $\chi \in \hat{H}_{1} \backslash U_{B}$ then $\left|S_{m}^{\prime}(\chi, 0)\right|$ $\leq C_{1} m B^{m-1}$ for any $m$. 
Proof. For each $\chi_{*} \in \hat{H}_{1}$ there exist open neighborhoods $U\left(\chi_{*}\right), W\left(\chi_{*}\right)$ of $\chi_{*}$ and continuous maps $h_{1}, \cdots, h_{d}: U\left(\chi_{*}\right) \rightarrow C^{d}$ such that

1) the closure of $W\left(\chi_{*}\right)$ is contained in $U\left(\chi_{*}\right)$.

2) for any $\chi \in U\left(\chi_{*}\right), h_{1}(\chi), \cdots, h_{d}(\chi)$ are linearly independent eigenvectors for $L(\chi, 0)$. By means of the Schmidt's orthogonalization we get a continuous map $P_{x_{*}}$ of $U\left(\chi_{*}\right)$ into the vector space Mat $(d, C)$ of $d$-dimensional complex matrices such that $P_{\chi_{*}}(\chi)^{-1} L(\chi, 0) P_{\chi_{*}}(\chi)$ is an upper triangular matrix for each $\chi \in U\left(\chi_{*}\right)$.

Since $\hat{H}_{1}$ is compact, one can choose finite $\chi_{1}, \cdots, \chi_{J} \in \hat{H}_{1}$ 'so that $\hat{H}_{1}=\cup_{j=1}^{J} W\left(\chi_{j}\right)$. Put $P_{j}=P \chi_{j}$ and define $Q_{j}(\chi, s)=P_{j}(\chi)^{-1} L(\chi, s) P_{j}(\chi)$ for $\chi \in U\left(\chi_{j}\right)$ and $s \in R$. We have

$$
\begin{aligned}
S_{m}^{\prime}(\chi, 0) & =m \operatorname{Trace}\left\{L^{\prime}(\chi, 0) L(\chi, 0)^{m-1}\right\} \\
& =m \operatorname{Trace}\left\{Q_{j}^{\prime}(\chi, 0) Q_{j}(\chi, 0)^{m-1}\right\} .
\end{aligned}
$$

Given $P \in \operatorname{Mat}(d, C)$ we shall denote by $P_{; k l}$ the $(k, l)$-entry of $P$. Put

$$
\begin{aligned}
C & =\max _{1 \leq j \leq J} \frac{\max }{\chi \in W\left(\chi_{j}\right)} \max _{k<l}\left|Q_{j}(\chi, 0)_{; k l}\right|, \\
C^{\prime} & =\max _{1 \leq j \leq J} \frac{\max }{\chi \in W\left(x_{j}\right)} \max _{k<l}\left|Q_{j}^{\prime}(\chi, 0)_{; k l}\right|,
\end{aligned}
$$

and

$$
N=\left[\begin{array}{rrrrrr}
0 & 1 & \cdot & \cdot & \cdot & 1 \\
\cdot & \cdot & \cdot & & & \cdot \\
\cdot & \cdot & \cdot & & \cdot \\
\cdot & & \cdot & \cdot & \cdot \\
\cdot & & & \cdot & 1 \\
0 & \cdot & \cdot & \cdot & \cdot & 0
\end{array}\right], \quad I=\left[\begin{array}{lllll}
1 & & & & \\
& \cdot & & & \\
& \cdot & & \\
& & \cdot & & \\
& & & \cdot & \\
& & & \cdot & \\
& & & & 1
\end{array}\right] \in \operatorname{Mat}(d, C)
$$

Since $N^{d+1}$ is a zero matrix and $\left|Q_{j}(\chi, 0)_{; k k}\right| \leq B$ if $\chi \in \hat{H}_{1} \backslash U_{B}$, there is a constant $C^{\prime \prime}$ such that

$$
\left|Q_{j}(\chi, 0)^{m-1}{ }_{; k l}\right| \leq(B I+C N)_{; k l}^{m-1} \leq C^{\prime \prime} B^{m-1}
$$

for each $\chi \in \hat{H}_{1} \backslash U_{B}$ and $k \leq l$. As $Q_{j}(\chi, 0)^{m-1}$ is an upper triangular matrix, we have

$$
\mid \text { Trace }\left\{Q_{j}^{\prime}(\chi, 0) Q_{j}(\chi, 0)^{m-1}\right\} \mid \leq \frac{1}{2} d(d+1) C^{\prime} C^{\prime \prime} B^{m-1},
$$

and get the conclusion.

Lemma 9. There exist positive constants $C_{2}, C_{3}, A$ and $B$ with $\sqrt{\lambda}$ $<B<A<\lambda$ such that if $\chi_{0}(-\alpha)=1$ (or if $\chi_{0}$ does not exist) 


$$
C_{2 m}(\alpha) \geq 2 m\left\{C_{2} A^{2 m-1}-C_{3} B^{2 m-1}\right\},
$$

and if $\chi_{0}(-\alpha)=-1$

$$
C_{2 m+1}(\alpha) \geq(2 m+1)\left\{C_{2} A^{2 m}-C_{3} B^{2 m}\right\}
$$

for any $m$.

Proof. Since $\lambda_{j}(\chi, s)$ varies continuously, one can find positive $\delta, B_{1}$ and $B_{2}$ so that

1) $\sqrt{\lambda}<B_{1}<B_{2}<\lambda$,

2) $\left|\lambda_{2}(\chi, s)\right|<B_{1}$ if $|s|<\delta$ and $\chi \in U_{B_{2}}$.

In particular, $\lambda_{1}(\chi, s)$ is a simple eigenvalue of $L(\chi, s)$, hence the map $\lambda_{1}(\chi, s)$ : $U_{B_{2}} \times(-\delta, \delta) \rightarrow C$ is smooth with respect to $s$. Here we note that $\lambda_{1}^{\prime}\left(\chi_{0}, 0\right)$ $=-\lambda_{1}^{\prime}(\mathbf{1}, 0)$ because $\lambda_{1}\left(\chi_{0}, s\right)=-\lambda_{1}(\mathbf{1}, s)$ for any $s$.

By Lemma 8, for any $B_{2}<B<\lambda$, we have $\left|S_{m}^{\prime}(x, 0)\right| \leq C_{1} m B^{m-1}$ for $\chi \in \hat{H}_{1} \backslash U_{B}$. Applying the same argument as Lemma 8, there is a constant $C$ such that

$$
\left|S_{m}^{\prime}(\chi, 0)-m \lambda_{1}^{\prime}(\chi, s) \lambda_{1}(\chi, s)^{m-1}\right| \leq C m B_{1}^{m-1}
$$

for any $m$ and $\chi \in \overline{U_{B}}$. In fact, as the eigenspace associated with $\lambda_{1}(\chi, s)$ varies smoothly with respect to $s$ and continuously with respect to $\chi$, we have a map $\tilde{L}(\chi, s): \quad U_{B} \times(\delta, \delta) \rightarrow \operatorname{Mat}(d-1, C)$ which is $s$-smooth and $\chi$-continuous, and which satisfies

$$
\operatorname{Trace}\left(\tilde{L}(\chi, s)^{m}\right)=S_{m}(\chi, s)-\lambda_{1}(\chi, s)^{m} .
$$

Now in the first place, we suppose $\chi_{0}(-\alpha)=1$. Since $\lambda_{1}^{\prime}(\mathbf{1}, 0)>0>$ $\lambda_{1}^{\prime}\left(\chi_{0}, 0\right)$, there is $B_{2}<B<\lambda$ such that

$$
\mathscr{R} e\left\{\chi(-\alpha) \lambda_{1}^{\prime}(\chi, 0)\right\}>0 \quad \text { for } \chi \in U_{B}^{+}
$$

and

$$
\mathscr{R} e\left\{\chi(-\alpha) \lambda_{1}^{\prime}(\chi, 0)\right\}<0 \quad \text { for } \chi \in U_{B}^{-} .
$$

Choose positive $A$ so that $B<A<\lambda$ and put $\Lambda=\min \left\{\mathscr{R}_{e}\left\{\chi(-\alpha) \lambda_{1}^{\prime}(\chi, 0)\right\} \mid \chi\right.$ $\left.\in \overline{U_{A}^{+}}\right\}$. Since $\mathscr{R} e\left\{\chi(-\alpha) \lambda_{1}^{\prime}(\chi, 0)\right\} \lambda_{1}(\chi, 0)^{2 m-1}>0$ for any $\chi \in U_{B}$, we have

$$
\begin{aligned}
C_{2 m}(\alpha) & =\int_{U_{B}} \mathscr{R} e\left\{\chi(-\alpha) S_{2 m}^{\prime}(\chi, 0)\right\} d \chi+\int_{\hat{H}_{1 \backslash U_{B}}} \mathscr{R} e\left\{\chi(-\alpha) S_{2 m}^{\prime}(\chi, 0)\right\} d \chi \\
& \geq 2 m \int_{U_{B}} \mathscr{R} e\left\{\chi(-\alpha) \lambda_{1}^{\prime}(\chi, 0) \lambda_{1}(\chi, 0)^{2 m-1}\right\} d \chi
\end{aligned}
$$




$$
\begin{aligned}
& -\int_{U_{B}}\left|S_{2 m}^{\prime}(\chi, 0)-2 m \lambda_{1}^{\prime}(\chi, 0) \lambda_{1}(\chi, 0)^{2 m-1}\right| d \chi \\
& -\int_{\hat{H}_{1 \backslash U} U_{B}}\left|S_{2 m}^{\prime}(\chi, 0)\right| d \chi \\
\geq & 2 m \int_{U_{A}^{+}} \mathscr{R} e\left\{\chi(-\alpha) \lambda_{1}^{\prime}(\chi, 0) \lambda_{1}(\chi, 0)^{2 m-1}\right\} d \chi \\
& -2 C m B_{1}^{2 m-1}-2 C_{1} m B^{2 m-1} \\
\geq & 2 m A^{2 m-1} \Lambda \operatorname{vol}\left(U_{A}^{+}\right)-2 m\left(C+C_{1}\right) B^{2 m-1} .
\end{aligned}
$$

(In case $\chi_{0}$ does not exist this argument can also be applied.)

In the second we suppose $\chi_{0}(-\alpha)=-1$. There is $B_{2}<B<\lambda$ with $\mathscr{R} e\left\{\chi(-\alpha) \lambda_{1}^{\prime}(\chi, 0)\right\}>0$ for any $\chi \in U_{B}$. Repeating the same computation we get the conclusion.

For $v \in V$ and $\alpha \in H_{1}(M, Z)$, let $\mathscr{C}_{n}(\alpha ; v)$ denote the set of $c \in \mathscr{C}_{n}(V ; \alpha)$ with $o(c)=v$. If $c \in \mathscr{C}_{n}(V ; \alpha)$ satisfies $g_{v}(c)>0$, then there exists $c^{\prime} \in$ $\mathscr{C}_{n}(\alpha ; v)$ which is equivalent to $c$. Note also that

$$
g_{v}(c) \exp f(c) \leq n \cdot \exp \left\{\left(\varepsilon-h_{\mathrm{top}}\right) n \cdot \min (\ell)\right\} .
$$

Therefore we have

Corollary 10. If $\chi_{0}(-\alpha)=1$, then

$$
\# \mathscr{C}_{2 m \nu}(\alpha ; v) \geq \frac{1}{2 m \nu}\left(C_{2} A^{2 m-1}-C_{3} B^{2 m-1}\right) \exp \left\{2\left(h_{\text {top }}-\varepsilon\right) m \nu \cdot \min (\ell)\right\}
$$

and if $\chi_{0}(-\alpha)=-1$, then

$\sharp \mathscr{C}_{(2 m+1) \nu}(\alpha ; v) \geq \frac{1}{(2 m+1) \nu}\left(C_{2} A^{2 m}-C_{3} B^{2 m}\right) \exp \left\{\left(h_{\mathrm{top}}-\varepsilon\right)(2 m+1) \nu \cdot \min (\ell)\right\}$.

In particular, $\mathscr{C}_{n}(\alpha ; v)$ is not empty for infinitely many $n$.

In order to get an estimate of $G_{m}(\alpha)$, we need auxiliary graphs (see [3] and [6]). Using informations on them, we can conclude the following by the same way as Lemmas 2-3 and 2-4 in [3].

Lemma 11. There exist positive constants $C_{4}$ and $C_{5}$ such that if $\chi_{0}(-\alpha)=1$

$$
G_{2 m}(\alpha) \geq 2 m\left\{C_{2} A^{2 m-1}-C_{3} B^{2 m-1}\right\}-8 C_{4} m^{3} \lambda^{m}-4 C_{5} m^{2},
$$

and if $\chi_{0}(-\alpha)=-1$

$$
G_{2 m+1}(\alpha) \geq(2 m+1)\left\{C_{2} A^{2 m}-C_{3} B^{2 m}\right\}-8 C_{4}(m+1)^{3} \lambda^{m+1}-4 C_{5}(m+1)^{2}
$$


for any $m \geq 1$.

This completes the proof of Proposition 5. In fact, since the right sides of (2) and (3) are dominated by $A^{2 m-1}$ and $A^{2 m}$ respectively, these estimates imply that $\sharp\left\{c \in \mathscr{P}_{2 m \nu}(V ; \alpha) \mid o(c)=v\right\}$ or $\sharp\left\{c \in \mathscr{P}_{(2 m+1) \nu}(V ; \alpha) \mid o(c)=v\right\}$ grows exponentially with respect to $m$.

Proof of Theorem 1. Given an open set $D$ in $U M$, we choose a Markov family $(V, E)$ for $\varphi_{t}$ with associated involution and of small size so that there is $v \in V$ with $v \subset D$. The previous argument implies that there is a prime closed path $c \in \cup_{k} \mathscr{C}_{k}(\alpha ; v)$ with prime $\mathfrak{p}=\Phi(\langle c\rangle)$. Since $\mathfrak{p} \cap v \neq$ $\varnothing$, this leads us to the conclusion.

\section{$\S 5$. Equidistribution of prime closed geodesics}

We now mention the equidistribution of closed geodesics with a preassigned homology class. It has been noted that there is a resemblance between prime closed geodesics and prime ideals in number fields ([9], [10], [17]). Our work in this section is an analogue of Chebotarev's density theorem and refines Bowen's result (cf. [8]). Throughout this section, let $H$ be a finite index subgroup of $H_{1}(M, Z)$ and $\mathscr{A} \in G=H_{1}(M, Z) / H$. We denote by $\hat{G}$ the character group of $G$. Given $\chi \in \hat{G}$ and a smooth function $g$ on $U M$, we define $\eta_{g}(s ; \chi)$ by

$$
\sum_{n=1 \mathfrak{p} ; \varphi t}^{\infty} \sum_{\text {closed orbit }} \chi(\llbracket \mathfrak{p} \rrbracket)^{n} \tau_{g}(\mathfrak{p}) h_{\text {top }} \exp \left\{-s n h_{\text {top }} \tau(\mathfrak{p})\right\},
$$

where

$$
\tau_{g}(\mathfrak{p})=\int_{0}^{\tau(\mathfrak{p})} g\left(\varphi_{t}(x)\right) d t, \quad x \in \mathfrak{p},
$$

If one defines a "weighted" $L$-function for $\varphi_{t}$ by

$$
\left.L_{g}(s, z ; \chi)=\exp \sum_{n=1}^{\infty} \frac{1}{n} \sum_{\mathfrak{p} ; \varphi_{t}-\text { closed orbit }} \chi(\llbracket \mathfrak{p}]\right)^{n} \exp \left\{-n h_{\mathrm{top}}\left(s \tau(\mathfrak{p})+z \tau_{g}(\mathfrak{p})\right)\right\}
$$

then

$$
\eta_{g}(s ; \chi)=-\frac{d}{d z} \log L_{g}(s, z ; \chi)_{z=0} .
$$

Analyzing these functions (see [2] and [8]), we have

Proposition 12. 1) $\eta_{g}(s ; \%)$ is analytic when $\mathscr{R} e s>1$.

2) $\eta_{g}(s ; 1)$ has an analytic extension to a neighborhood of $\mathscr{R} e s>1$ 
except for a simple pole at $s=1$ of residue $\int g d \mu$.

3) If $\chi \neq 1, \eta_{g}(s ; \chi)$ is analytic in some neighborhood of $\mathscr{R}_{e} s \geq 1$.

For each $\mathscr{A} \in G$, we set

$$
\begin{aligned}
F_{g}(\mathscr{A}, T) & =\frac{1}{\sharp G} \sum_{\mathfrak{p}} \tau_{g}(\mathfrak{p}) h_{\mathrm{top}}, \\
\pi_{g}(\mathscr{A}, T) & =\frac{1}{\sharp G} \sum_{\mathfrak{p}} \tau_{g}(\mathfrak{p}) / \tau(\mathfrak{p}),
\end{aligned}
$$

where in the summations $\mathfrak{p}$ runs over all $\varphi_{t}$-orbit cycles with $[p(\mathfrak{p})] \in \mathscr{A}$ and $\tau(\mathfrak{p}) \leq(\log T) / h_{\text {top }}$. Since

$$
\int_{1}^{\infty} T^{-s} d F_{g}(\mathscr{A}, T)=\frac{1}{\sharp G} \sum_{x \in \hat{G}} \chi(-\mathscr{A}) \eta_{g}(s ; \chi),
$$

Tauberian theorem implies that

$$
F_{g}(\mathscr{A}, T) / T \longrightarrow \frac{1}{\# G} \int g d \mu .
$$

We can also conclude $\pi_{g}(\mathscr{A}, T) \log T \sim F_{g}(\mathscr{A}, T)$. Therefore

$$
\begin{aligned}
\hat{\mu}_{T, \varepsilon, \mathscr{A}}(g) & =\pi_{g}\left(\mathscr{A}, \exp (T+\varepsilon) h_{\mathrm{top}}\right)-\pi_{g}\left(\mathscr{A}, \exp (T-\varepsilon) h_{\mathrm{top}}\right) \\
& \sim\left\{h_{\mathrm{top}} T \sharp G\right\}^{-1} \exp \left(h_{\mathrm{top}} T\right) \int_{U M} g d \mu\left\{\exp \left(\varepsilon h_{\mathrm{top}}\right)-\exp \left(-\varepsilon h_{\mathrm{top}}\right)\right\},
\end{aligned}
$$

hence

$$
\mu_{T, \varepsilon, \mathscr{A}}(g) \longrightarrow \int_{U M} g d \mu \text { and } \mu_{T, \varepsilon, \mathscr{A}}(g) / \mu_{T, \varepsilon, \mathscr{P}}(g) \longrightarrow 1
$$

for any $\mathscr{A}, \mathscr{B} \in G$.

We now examine the measure $\nu_{T, \varepsilon, \AA}$. Since

$$
\sum \chi(\llbracket \mathfrak{p} \rrbracket) \tau_{g}(\mathfrak{p}) h_{\mathrm{top}} \exp \left\{-n s h_{\mathrm{top}} \tau(\mathfrak{p})\right\}
$$

contains the essential part of $\eta_{g}(s ; \chi)$, one obtains

$$
h_{\mathrm{top}} \sum_{\mathfrak{p} ; \tau(\mathfrak{p}) \leq T} \tau_{g}(\mathfrak{p}) \sim \exp \left(h_{\mathrm{top}} T\right) \int g d \mu,
$$

which leads us to Theorem 2.

\section{ReFerences}

[1] T. Adachi, Markov families for Anosov flows with an involutive action, Nagoya 
Math. J., 104 (1986), 55-62.

[2] T. Adachi and T. Sunada, Twisted Perron-Frobenius Theorem and L-functions, J. Funct. Anal., 71 (1987), 1-46.

[ 3 ] - Homology of closed geodesics in a negatively curved manifold, J. Diff. Geom., 26 (1987), 81-99.

[4] R. Bowen, Periodic orbits for hyperbolic flows, Amer. J. Math., 94 (1972), 1-30.

[5] - The equidistribution of closed geodesics, Amer. J. Math., 94 (1972), 413-423.

[6 ] - Symbolic dynamics for hyperbolic flows, Amer. J. Math., 95 (1973), 429-460.

[ 7 ] P. Eberlein, When is a geodesic flow Anosov type? I, J. Differential Geom., 8 (1973), 437-463.

[8] W. Parry, Bowen's equidistribution theory and the Dirichlet density theorem, Ergodic Theory Dynamical Systems, 4 (1984), 117-134.

[ 9 ] W. Parry and M. Pollicott, An analogue of the prime number theorem for closed orbits of Axiom $A$ flows, Ann. of Math., 118 (1983), 573-591.

[10] - The Chebotarev theorem for Galois coverings of Axiom $A$ flows, preprint (1984), Warwick Univ.

[11] J. F. Plante, Homology of closed orbits of Anosov flows, Proc. Amer. Math. Soc., 37 (1973), 297-300.

[12] M. Pollicott, Meromorphic extension of generalised zeta function, Invent. math., 85 (1986), 147-164.

[13] —- A note on uniform distribution for primes and closed orbits, preprint.

[14] M. Rees, Checking ergodicity for some geodesic flows with infinite Gibbs measure, Ergodic Theory Dynamical Systems, 1 (1981), 107-133.

[15] D. Ruelle, Thermodynamic Formalism. Addison-Wesley, 1978.

[16] T. Sunada, Geodesic flows and geodesic random walks. Advanced Studies in Pure Math., 3 (Geometry of Geodesics and Related Topics) 1984, 47-86.

[17] — - Tchebotarev's density theorem for closed geodesics in a compact locally symmetric space of negative curvature, preprint.

Department of Mathematics

Nagoya University,

Chikusa-ku Nagoya

464 Japan

Current address:

Department of Mathematics

College of General Education

Kumamoto University

Kumamoto, 860 Japan 